\title{
EL CANON LITERARIO EN AMÉRICA LATINA
}

\author{
Genara PULIDO TIRADO
}

Universidad de Jaén

gpulido@ujaen.es

\begin{abstract}
Resumen: La consideración del canon literario había ocupado un lugar importante en los estudios literarios latinoamericanos desde el momento en que surgió la necesidad de definir la especificidad de la propia literatura y de las culturas literarias de este vasto espacio geográfico y cultural, hecho ligado siempre a antiguas y nuevas dependencias de carácter político y cultural. La teorización en este campo, que se produce a finales del siglo Xx y principios del siglo XXI con fuerza en Norteamérica y Europa Occidental, se da también en distintos puntos de América Latina. En este caso se trata de determinar qué es el canon y de poner de manifiesto la necesidad de revisar un canon de carácter eurocéntrico que ignoraba manifestaciones importantes de la literatura latinoamericana como la oral, indígena, negra, popular o mestiza. Al citado proceso dedicamos el presente estudio.
\end{abstract}

Abstract: The consideration of the literary canon has occupied an important place in Latin American studies from the moment in which the need arose to define the specificness of the literature itself and literary cultures belonging to this vast geographical and cultural domain, a fact always linked to old and 
new dependencies of a political and cultural character. The theorization in this field, which effectively took place at the end of the nineteenth and the beginning of the twentieth centuries in North America and Western Europe also occurred in several places in Latin America. In this last instance an important issue is to determine what the canon is and the need to revise a canon of a Eurocentric character which has ignored important manifestations of Latin American literature such as the oral, indigenous, black, popular or mestizo traditions. This study is centred on analysing the aforementioned process.

Palabras clave: Canon literario. Latinoamérica. Estudios literarios. Teoría de la literatura. Literatura culta. Literatura oral. Literatura indígena. Literatura negra. Literatura mestiza.

Key Words: Literary canon. Latin America. Literary studies. Literary theory. Highbrow literature. Oral literature. Indigenous literature. Black literature. Mestizo literature.

La confrontación entre partidarios del formalismo y partidarios del compromiso constituye un episodio fundamental de la crítica literaria latinoamericana del siglo xx. Sin embargo, cuando la nueva novela hispanoamericana se está dando a conocer en el mundo se detectan consecuencias importantes en el ámbito del canon literario ya que cada tendencia lleva a cabo una concreta interpretación de las figuras estelares. «En última instancia se debate la apropiación de los autores, la significación de la lectura de determinados textos y, a través de ellos y algunos sectores, lo que esas mismas lecturas hacen a la comprensión más amplia del mundo al que remiten», como ha resumido puntualmente Sosnowski (1987: 149). El auge de la nueva literatura latinoamericana y su reconocimiento internacional están interrelacionados y remiten a factores muy concretos como la desvinculación de muchos escritores de la Revolución cubana tras el escándalo del «caso Padilla ${ }^{1}$ o el exilio voluntario o involuntario de autores cubanos y del Cono Sur tras el surgimiento de regímenes totalitarios. El reconocimiento internacional, a su vez, tiene su propio canon ya que, respetando a los autores reconocidos en el canon académico como Martí, Darío, Vallejo, Huidobro o

\footnotetext{
${ }^{1}$ Sobre el caso Padilla, ver, entre otros, Manuel Díaz Martínez (s.f.).
} 
Neruda, de los narradores se destacan aquellos que internacionalizan la nueva novela: Borges, Cortázar, Onetti, Donoso, Vargas Llosa, García Márquez, Rulfo, Fuentes, Paz, Carpentier, Cabrera Infante y Roa Bastos encabezan las nóminas. Dicho canon conlleva una descompensación, lo que se manifiesta en una serie de hechos:

Se sigue leyendo una literatura de epígonos; se plantean cortes temáticos transversales; se periodiza con la arbitrariedad de los números y las edades las generaciones literarias, y se fundan versiones parciales de la producción literaria que tienden a desconocer un factor central: que los relojes culturales de las diversas regiones y áreas latinoamericanas no están sincronizados [...] Al optar, por ejemplo, por la experimentación literaria como criterio de selección, quedan fuera vastas regiones cuya respuesta literaria está adecuadamente servida por las tendencias que fueron abandonadas en zonas cosmopolitas hace varias décadas. Al concentrar la lectura en la producción urbana (segmento por cierto válido) se presupone una serie de interrogantes sobre el sentido de esa producción desde su grado de diferencia con un balance de los textos que constituyen su base local, en la medida en que toda selección presupone la capacidad de ese texto de representar un segmento que puede excederlo, concentrarse sistemáticamente en sólo algunas de las variantes de la literatura hispanoamericana —el embate experimental, por ejemplo - contribuye no sólo a una distorsión del amplio texto literario sino también del mundo del cual surgen esas páginas (Sosnowski, 1987: 151$152)^{2}$.

Una de las consecuencias más directas de la revisión del canon ha sido la publicación en las últimas décadas del siglo Xx de importantes estudios sobre la literatura colonial, de cronistas — se trata de explorar las etapas fundacionales del imaginario americano- y de figuras importantes del barroco - las polémicas entre al barroco y el neobarroco siempre han puesto de manifiesto el difícil dilema que se produce a nivel crítico cuando se trata de reconocer la deuda con el poder colonial y reivindicar lo propio como específico-. Todo ello ha conducido, además, al debate sobre las literaturas nacionales y a la reivindicación y estudio de la cultura y la literatura de diversas comunidades indígenas, negras o mestizas.

$\mathrm{Al}$ sacar el concepto de lo literario del ámbito restringido de los textos supuestamente dotados de unas cualidades estéticas exclusivas para reconocer, como mínimo, que el texto literario no es un ente aislado en tanto que está en

\footnotetext{
${ }^{2}$ Sobre la literatura del boom y el canon, ver también López de Abiada y Pérez Cino (2005).
} 
permanente contacto con otros discursos y formas culturales, se altera la visión tradicional de los géneros literarios. En primer término, se produce la irrupción en el campo de estudio de manifestaciones desdeñadas hasta época reciente como las fotonovelas, las revistas, las tiras cómicas, el cine, la música y los bailes populares, y otros. Por otra parte, la tríada genérica tradicional se ve renovada en tanto que la narrativa presenta nuevas características y nuevas manifestaciones - como el cuento o el microrrelato-, la poesía se manifiesta tanto en su vertiente lírica como prosaica, y el teatro asiste a una serie de cambios que lo convierten en una manifestación postmoderna por excelencia puesto que se adapta al apasionante mundo del espectáculo que desplaza una expresión viva como es el teatro de la cárcel del texto (el teatro colectivo o el teatro campesino son ejemplos paradigmáticos al respecto). Pero no voy a entrar aquí en vicisitudes relativas a géneros literarios concretos, pues el cuestionamiento del canon como fenómeno general y determinante de la literatura y los estudios literarios latinoamericanos tiene en sí mismo una entidad que es preciso desentrañar antes de entrar en las derivaciones de este fenómeno que ha provocado no pocos cambios en la literatura occidental de las últimas décadas.

Ni que decir tiene que la entrada en escena de la cultura en el ámbito de los estudios literarios es un factor que actúa de forma decisiva en el cuestionamiento del canon literario latinoamericano. Este cuestionamiento es positivo siempre que se actúa con criterios y no de forma indiscriminada reivindicando todo lo marginado por las instituciones y la crítica literaria y académica por el mero hecho de haberlo sido y no por sus valores estético-literarios. Los estudios sobre el canon se pueden dividir en tres ramas: los descanonizadores, esto es, aquellos que han pretendido desmitificar obras concretas o bien la institución literaria misma; los canonizadores, que son los que han llamado la atención sobre manifestaciones olvidadas como las literaturas regionales, indígenas, negras..., o escritas por mujeres; y estudios sobre el canon actual ya que, una vez que se ha cobrado conciencia de que todo canon es una imposición social generada por un grupo dominante y por intereses ideológicos concretos, la responsabilidad del estudio de la literatura aumenta de forma considerable.

En América Latina el interés por esta cuestión tiene unas raíces hondas puesto que la dependencia colonial conlleva el cuestionamiento de los modelos canónicos que se imponen desde la metrópoli, los cuales, ya en un primer momento, aplican violentamente un modelo supuestamente universal (europeo) despreciando la tradición oral de las literaturas indígenas. Pero, por otra parte, hay que destacar que dentro del mismo subcontinente se producen 
marginaciones que tardarán en ser rectificadas como el abandono y posterior reconocimiento de la literatura de Centroamérica. Cuando se empieza a hablar de crisis de la crítica literaria se comienza a contemplar también la crisis del concepto de literatura. Carlos Rincón, tempranamente - recordemos que en Estados Unidos la generalización y el tratamiento como «moda» de la problemática del canon se produce en la década de los noventa, aunque se retrotrae a los sesenta-, anota cambios determinantes en este punto:

Visto desde una perspectiva general, lo decisivo del proceso en que se hallan inscritas nuestras letras, de su dinámica, no reside entonces en el surgimiento de una nueva novelística. Así tal novelística haya conseguido no solamente disolver la distancia épica, transformar su materia, sus métodos y, por esa vía, sus funciones, sino constituirse, dentro del proceso histórico social y literario, en el género dominante, de tal manera que los demás géneros se han visto tocados por exigencias de narratividad que les dan otro sentido y acento y los ligan de manera más íntima al presente. [...] El fenómeno es otro: la presión del proceso social en el continente ha llevado, a nivel ideológico, no sólo a hacer saltar los marcos, sino a ponerse en cuestión la realidad misma del espejismo de esa esencia sustancialista de la literatura vital para que ésta mantenga su estatus tradicional (Rincón, 1978: 17)

Considerada la literatura como producto histórico, como praxis social, tal como hace Rincón, estos cambios no constituyen objeto de alarma puesto que lo que le corresponde al crítico es analizar la situación histórico-social en la que se producen esos cambios. La nueva literatura que surge de este contexto exige una nueva relación con el lector debido a que ya no se considera que el fenómeno literario esté constituido única y exclusivamente por el lenguaje; en este punto debemos recordar que Rincón se formó con el romanista Werner Krauss en la Universidad de Leipzig, por lo que conocía la estética de la recepción, de la que tomó algunos conceptos y a la que criticó también.

Walter Mignolo, latinoamericano afincado en Estados Unidos, ha abordado la cuestión del canon de forma exhaustiva y en relación a América Latina. Partiendo de la idea de que una de las funciones principales de la formación del canon, literario o no, es asegurar la estabilidad y adaptabilidad de una comunidad de creyentes, considera oportuno diferenciar, cuando el problema del canon se relaciona con actividades disciplinarias, los aspectos vocacionales y los aspectos epistémicos o disciplinarios de la formación del canon. En Latinoamérica el canon oficial se forma basándose en la lengua y en los valores de las culturas colonizadoras más importantes, la espa- 
ñola y la portuguesa, e ignorando las culturas amerindias, lo que se puede observar fácilmente en las primeras historias literarias del subcontinente americano. Ahora bien, los debates sobre la formación del canon se desarrollan en tres niveles:

A nivel vocacional, un canon literario debería verse en el contexto académico (¿qué debería enseñarse y por qué?). A nivel epistémico, la formación del canon debería analizarse en el contexto de los programas de investigación, como un fenómeno que debe ser descrito y explicado (¿cómo se forman y se transforman los cánones?, ¿qué grupos o clases sociales esconde el canon?, etc.). A nivel de las fronteras culturales, un canon debería considerarse como relativo a la comunidad y no como una relación jerárquica respecto a un canon fundamental, ni tampoco dentro de un modelo evolutivo en que los ejemplos canónicos se convierten en el paraíso al que aspiran las literaturas y en medida de la organización jerárquica (Mignolo, 1991: 145-146).

Diferenciar estos niveles es importante ya que deben permitirnos evitar proyectar los valores del llamado primer mundo sobre el llamado tercer mundo así como minusvalorar los criterios del tercer mundo comparándolos con los del primero, lo que se puede lograr acudiendo a descripciones epistémicas de la literatura que puedan diferenciarse claramente de las definiciones vocacionales. La literatura, desde esta perspectiva, debería entenderse como una práctica discursiva regional, y la formación y transformación del canon como un subsistema dentro del sistema.

La enseñanza de la literatura del tercer mundo (América Latina) que se da en el primer mundo (Estados Unidos) se produce en términos de colonizado y colonizador, hecho que sólo puede corregirse reconociendo que existen otras alternativas a la integración de la periferia en el centro y que enseñar la habilidad de leer es distinto a la habilidad de leer un conjunto de textos canónicos, esto es, textos seleccionados por ser portadores de determinados valores estéticos, étnicos o tradicionales.

Entendida la formación del canon en los estudios literarios como una manifestación de «la necesidad de las comunidades humanas de estabilizar su pasado, adaptarse al presente y proyectar su futuro» (Mignolo, 1991: 251), nos seguimos encontrando con el hecho de que en una sociedad plural el canon, para los que representan el poder, es distinto al canon de las comunidades marginadas. Pero la crítica literaria debería estudiar no sólo la vertiente epistémica del canon, sino también la vocacional, lo que conduciría a sustituir los problemas normativos relativos a la formación y transformación del canon 
por explicaciones que contemplen las condiciones en las que se forman y transmiten los cánones, esto es, «Preguntas como quién decide por quién y por qué debería leerse un grupo de textos determinado tomarán el lugar de preguntas como qué se debería leer» (Mignolo, 1991: 256; la cursiva es de W.M.). La problemática que se establece entre un canon literario y otras literaturas conduce al cuestionamiento mismo del concepto de literatura y, al final, también del canon, esto es, primero, la necesidad de incluir la literatura no occidental, del tercer mundo, de mujeres, etc., en el canon pone de manifiesto la existencia de un grupo de investigadores que a nivel vocacional sienten la necesidad de que el canon se transforme para representar a una población plural como la americana; y, segundo, desde un punto de vista vocacional esta ampliación del canon se entiende y se permite, pero desde un punto de vista epistémico no resulta lógico. En definitiva, «a nivel vocacional, debemos hablar sobre $e l$ canon (dando por descontado que "el" equivale a "nuestro"). Sin embargo, a nivel epistémico, deberíamos darnos cuenta de que existen tantos cánones como comunidades» (Mignolo, 1991: 265).

Pocos años después Mignolo vuelve a abordar el tema atendiendo ahora al auge de los estudios sobre la cultura, en los que se produce un proceso parecido al que se detecta en los estudios literarios: las «esencias» culturales no están representadas por un canon, sino que son «creadas» y mantenidas por él. A la preocupación por la noción de lo literario se une la del límite de la noción de lo latinoamericano, puesto que Latinoamérica sobrepasa la América Hispana y Portuguesa e incluye el Caribe español, francés e inglés, e incorpora también las literaturas fronterizas de Estados Unidos escritas tanto en español como en inglés ${ }^{3}$. En cuanto a la literatura, no puede entenderse ya según viejas concepciones de orden estético o estructural puesto que «el campo de los estudios literarios se concibe más como un corpus heterogéneo de prácticas discursivas y de artefactos culturales» (Mignolo, 1994-1995: 25). Algunas de las consecuencias de esta ampliación de horizontes han sido señaladas por el mismo Mignolo:

\footnotetext{
3 Juan Poblete ha estudiado la literatura latina en Estados Unidos y ha trabajado con dos hipótesis: primera, se trata de textos que obligan a la literatura estadounidense y a la latinoamericana a enfrentarse a su carácter de formación literaria heterogénea y, por consiguiente, a aceptar la traducción cultural y lingüística; segunda, este tipo de discursos expone una transformación posible del sentido de lo nacional literario y sus formas de territorialización del espacio y la cultura nacional a la par que pone de manifiesto una renovación de su potencial crítico. Se trata, en todo caso, de una literatura emergente que busca un lugar propio en medio de un espacio lleno de interrogantes (ver Poblete, 2003, 2006 y Poblete, coord., 2005). Alberto Sandoval y Frances Aparicio coordinaron en 2005 un número monográfico de la Revista Iberoamericana sobre Hibridismos culturales: la literatura y la cultura de los latinos en los Estados Uni$d o s$, que aporta numerosas e importantes perspectivas sobre el tema.
} 
La apertura del campo de estudios del canon al corpus trajo dos consecuencias ligadas, la una, a la diversidad de prácticas discursivas involucradas en el corpus y, la otra, ligada a la diversidad lingüística de América Latina y a la movilidad social que produjo zonas fronterizas y productos lingüística y culturalmente híbridos, como es el caso de la literatura «latina» en Estados Unidos. La primera se manifestó en estudios interdisciplinarios, en los que los literaturólogos crearon alianzas con antropólogos, historiadores y socio-lingüistas, fundamentalmente. La segunda se manifestó en un paulatino crecimiento del interés por los estudios comparativos (Mignolo, 1994-1995: 29).

La rebelión contra un canon heredado de la colonia ha tenido distintas manifestaciones en tanto que diferentes investigadores han llamado la atención sobre elementos excluidos arbitrariamente de ese canon. Así, Ángel Rama, al hablar en su Ciudad letrada (1984) de la violencia con que los colonizadores imponen la letra frente a la voz, idea que desarrolla Martin Lienhard en La voz y su huella. Escritura y conflicto étnico-social en América Latina (1991), donde a los estudios de distintas prácticas discursivas, orales y escritas, une el análisis de la obra de Arguedas, autor bilingüe y bicultural para mostrar que existen múltiples manifestaciones en el canon de las prácticas discursivas coloniales que destacan por su carácter plurilingüe y multicultural y que llegan hasta el presente. De aquí se deduce que si existen identidades coexistentes también pueden existir cánones que coexisten, por lo que las relaciones entre el canon y el corpus se reconfiguran:

Mientras el canon pareciera implicar una relación de tipo sustancial entre prácticas y paradigmas culturales, el corpus necesitaría sólo de una manejable delimitación espacial y temporal. Mientras el canon implica cuestiones de identidad (¿qué es lo latinoamericano?), el corpus necesita de parámetros locativos (¿dónde y cuándo se relacionaron las prácticas discursivas en cuestión?). No obstante, la posibilidad de pensar en cánones paralelos, coexistentes y mutuamente alternativos incluye una movilidad del canon en el corpus que depende, en última instancia, de las identidades individuales y grupales y del poder ejercido por los sujetos del discurso y la institución que los apoya y los promueve en el espacio social (Mignolo, 1994-1995: 29).

Jean Franco anota a principios de los ochenta la íntima relación existente entre la crisis de los estudios literarios, la reevaluación de la crítica cultural y el cambio de concepto de lo literario:

La crítica literaria no es lo que era, en parte porque un grupo heterogéneo de filósofos, antropólogos y pensadores políticos (Derrida, Foucault, Althusser y Lévi-Strauss) han afectado sus categorías básicas, y en parte también porque 
la lectura de textos dirigidos a entender el cómo de su significado, es hoy del interés lo mismo para los filósofos, políticos, antropólogos e historiadores que para los críticos literarios. De hecho estamos viviendo la emergencia de una crítica cultural y un análisis del discurso que van mucho más allá del estudio y evaluación de un pequeño número de textos literarios canonizados y que cuestiona las razones por las que ciertos textos llegan a ser evaluados como «literarios» (Franco, 1981: 7).

Para esta investigadora, que se inscribe a sí misma en una nueva sociocrítica, las áreas a las que hay que atender y que cobrarán gran notoriedad a partir de los cambios señalados son tres: 1) el análisis de la vida cotidiana, que implica el estudio del control del estado y la disciplina familiar, actividades que se realizan en el tiempo libre, modas en el vestir o conducta; 2) el análisis de los medios con la consecuente consideración de las diferencias existentes entre una cultura oral y otra basada en nuevas tecnologías de la comunicación que producen nuevos productos (fotonovela, folletín, etc.) acorde con la ideología del capitalismo avanzado que no se pueden ignorar por cuanto nos enseñan que el concepto de lo literario es histórico y por tanto puede cambiar a lo largo de la historia; 3) y la crítica feminista, poco desarrollada entonces en Latinoamérica, a cuyo desarrollo contribuirá notablemente Jean Franco — su libro de 1989 Plotting Women, en el que estudia las implicaciones del género discursivo por lo que tiene de fundamental en la lucha de la mujer por el poder discursivo, tomando a Méjico como punto de referencia, marca toda una línea de investigación-. En la visión de esta autora no se trata de que la crítica literaria sea desplazada por un determinado tipo de estudios sociológicos, culturales o antropológicos, sino que se vea enriquecida al ampliar su campo de estudio para poder intervenir en la investigación social con total legitimidad.

En un ámbito geográfico y cultural como el latinoamericano, marcado por la pluralidad de manifestaciones literarias y culturales, la publicación del libro de Bloom, El canon occidental (1994), constituyó un motivo excelente para centrar las reflexiones que se estaban produciendo sobre este tema. En 1998 Susana Cella edita un volumen colectivo, Dominios de la literatura: acerca del canon, en el que pone a repensar la cuestión a destacados críticos de la zona. En el año 2002, en la revista virtual de la Universidad de Chile Cyber Humanitas, J. M. Silva publicaba un trabajo amplio en el que se revisaba el concepto de canon, las versiones de ese concepto, su operatividad en el ámbito literario, así como su manifestación plural en Latinoamérica ${ }^{4}$.

${ }^{4}$ Sobre el canon en Latinoamérica pueden verse distintos trabajos en <http://www.monografias.com/ trabajos13/canon/canon.sht>. 
También en el año 2002 publica su tesis doctoral (para obtener el grado de Licenciado en Literatura en la Universidad de Puebla) sobre el tema Ignacio M. Sánchez-Prado, El canon y sus formas: la reinvención de Harold Bloom y sus lecturas hispanoamericanas.

Frente a las expectativas que despierta en España la obra de Bloom ${ }^{5}$, en Hispanoamérica, quizás porque la preocupación por el canon era, como se ha dicho ya, anterior, la obra de Bloom se contempla como una oportunidad de repensar cuestiones que afectan muy directamente a la literatura y la cultura latinoamericanas ${ }^{6}$, esto es, aunque no se esté a favor de la fijación de un canon a la manera propuesta por Bloom, el hecho de que éste plantee la situación es una confirmación venida de la metrópoli de que la literatura que pasa a la historia lo hace no por méritos intrínsecamente literarios, sino por cuestiones históricas en las que la política cultural y la ideología política desempeñan una función determinante.

La obra de Sánchez-Prado es un estudio sobre la trayectoria de Harold Bloom, que se centra en su visión del canon y contempla las aplicaciones de este concepto a Latinoamérica. En este ámbito plural, como en pocos, el canon se forma sobre la noción de «literatura culta» —e ignora, por tanto, las literaturas indígenas, la tradición oral, la cultura popular y manifestaciones literarias escritas no consideradas cultas-, noción lógicamente heredada de la colonia. La fundación de este canon se produce, a juicio de Sánchez-Prado, en la llamada «Generación del Ateneo», cuyos principales representantes son Alfonso Reyes y Pedro Henríquez Ureña, autores que, inscritos ambos en la escuela del americanismo literario, pretenden definir una identidad regional en la que el lector hispanoamericano pueda ver reflejadas sus propias inquietudes. En la siguiente generación, Roberto Fernández Retamar reivindica la autonomía cultural y la especificidad literaria como resultado de una especificidad histórica y la literatura como una práctica ideológica relacionada con la constitución de la cultura continental. Ángel Rama, al cuestionar la cultura letrada y reivindicar la transculturalidad como elemento definidor de la narrativa latinoamericana, da un vuelco al viejo concepto de canon. El

\footnotetext{
5 Para su recepción, ver Pulido Tirado (1999).

${ }^{6}$ En la reseña de la obra de Bloom realizada por Christopher Domínguez Michael en 1997 el autor no se limita a dar noticia del contenido de la obra, también juzga a Bloom, al que califica de «crítico conservador», «patriarca de Yale», «hombre de letras liberal» que llamó «"pandilla de idiotas" a los críticos españoles que protestaron contra la Leyenda Negra que enturbia El canon occidental» (Domínguez Michael, 1997: 58) por lo que, como lector anglosajón típico, no resulta extraño que crea que con Cervantes basta y, en consecuencia, la atención prestada a la literatura que llama «hispano-portuguesa» sea insuficiente y cuestionable.
} 
concepto de heterogeneidad cultural de Antonio Cornejo Polar y sus estudios de las literaturas indígenas son decisivos también en este sentido. De todo este proceso se desprende una serie de hechos que señala Sánchez-Prado:

En años más recientes, y quizá como consecuencia de lecturas de la obra de Rama y de Cornejo Polar desde una perspectiva postmoderna, el concepto de canon ha sido objeto de una revisión constante. Sin embargo, las discusiones y a no han girado en torno a nuevas maneras de constituir un canon, sino que se ha puesto en entredicho aún incluso la posibilidad de que éste exista en realidad o tenga una validez más allá de su función como legitimador de un proyecto cultural de élites ilustradas. Esos cuestionamientos tienen dos fuentes teóricas, que van desde la incorporación de los estudios culturales y postcolonialistas al ámbito de la literatura latinoamericana hasta la reevaluación de géneros literarios poco tradicionales, como el testimonio (Sánchez-Prado, 2002: 210).

$\mathrm{Ni}$ que decir tiene que estos cambios operados no son del agrado del joven investigador, que convencido de que el canon es una de las formas que tiene la cultura de representarse a sí misma, cree que el canon no debe verse como imposición, sino como un elemento que nos ofrecen las culturas para que podamos estudiarlas y comprenderlas, por lo que no puede ser eliminado. Sánchez Prado elogia el carácter elitista que confiere Bloom al canon, lo que le lleva a rechazar cualquier uso del canon como instrumento extraliterario. El diagnóstico de Bloom, además, se califica de «exacto» (SánchezPrado, 2002: 263) en el sentido de que los cánones y contracánones utilizan la literatura para enmascarar intereses políticos y académicos que se plantean detrás del privilegio de que gozan algunas manifestaciones culturales y sus formas de estudio (aunque no se aluda a la cultura y a los estudios sobre la cultura, la presencia de éstos es obvia). En suma,

Para este análisis [el del canon en América Latina], el libro de Bloom es un ejemplo ideal por dos motivos. Primero, es una propuesta de canon atípica, puesto que busca librarse de las implicaciones ideológicas que este término conlleva comúnmente, por lo que permite problematizar las preguntas que planteamos en torno al tema. Segundo, este libro suscitó respuestas en el ámbito latinoamericano, las cuales se inscriben en términos que corresponden a dos manifestaciones diferentes de los estudios latinoamericanos que no provienen del paradigma generado por la academia estadounidense o por las aproximaciones tradicionales al tema (Sánchez Prado, 2002: 269).

¿A qué respuestas se refiere este autor? Una de las primeras y más significativas es la recopilación de trabajos ya citada que publica Susana Cella 
en Buenos Aires en 1998, Dominios de la literatura: acerca del canon, libro que se organiza en torno a tres secciones temáticas: «El devenir de una palabra», en la que se estudia el concepto e implicaciones de la teoría de Bloom; «El ámbito continental», en la que se pretende ver las consecuencia de la aplicación del concepto a América Latina; y «Canon y literatura nacional», dedicada sólo a escritores argentinos. Los trabajos críticos no habían sido elaborados «a propósito y en relación exclusiva» al libro de Bloom; están acompañados, además, de fragmentos de obras literarias que ilustran las tesis que se defienden y se recoge una encuesta realizada a cincuenta escritores con el objetivo de que eligieran las diez novelas argentinas más importantes a su juicio. El propósito es demostrar, primero, que el tema del canon existía antes de Bloom ${ }^{7} \mathrm{y}$, segundo, que afecta, por un lado, a ámbitos regionales $\mathrm{y}$, por otro, universales, que están al margen del anglocentrismo bloomiano, de ahí que la consideración de un elevado número de teóricos y pensadores (Auerbach, Eco, Kermode, Calvino, Freud, Adorno, Benjamín, Rodríguez Monegal, etc.) en relación con el tema cobre sentido en tanto que pone de manifiesto la complejidad de la cuestión. Si bien es cierto que la obra no da soluciones ni visiones programáticas en torno a la problemática, no se puede negar el carácter inquietante que se desprende de una colección desigual de escritos que bucean en el complejo campo de los estudios literarios latinoamericanos del siglo Xx. En relación al canon y la literatura latinoamericana la observación de Zanetti es importante:

Justamente uno de los problemas del canon latinoamericano es que más bien se afianza débilmente, dado el carácter errático de las lecturas y relecturas, la carencia de interpretaciones críticas reiteradas, así como la ausencia de una suerte de Academia supranacional que se plante como voz autorizada, cuyos dictámenes pondrían a prueba las nuevas generaciones de lectores. Las discusiones suelen rebasar poco el nivel nacional, salvo en las polémicas muy acotadas a situaciones concretas y a ciertos autores (Zanetti, 1998: 92).

Ni que decir tiene que tal visión es rechazada por Sánchez-Prado. ¿Puede referirse Sánchez-Prado, cuando habla de un acercamiento que se aleja de

${ }^{7}$ En el capítulo que abre la obra Cella se expresa con suma claridad: «Podría pensarse entonces que esta reaparición del "canon", objeto perdido o imaginario de la crítica, como síntoma determinado por un entrecruzamiento de series que van desde una idea de la literatura -lenguaje, arte, modulación de imaginarios, testimonio de saberes o hechos, vía privilegiada de conocimiento, thesaurus o biblioteca infinita - hasta formas de apropiación de espacios sociales, institucionales y políticos. En esta casi absoluta enumeración caben las manifestaciones que han tenido y tienen lugar en lo que a políticas literarias se refiere» (Cella, 1998: 7-8). 
las aproximaciones tradicionales, al tema a las aportaciones de autores como Ángel Rama o Cornejo Polar? Tampoco:

\begin{abstract}
Ángel Rama y Cornejo Polar hacen las propuestas que permiten el planteamiento de un canon no impositivo en el ámbito latinoamericano. La apertura del canon que significan las nociones de transculturación ${ }^{8}$ y heterogeneidad ${ }^{9}$ hacen que el canon ya no sea la representación cultural de un grupo hegemónico, sino que se plantea esta apertura a partir de la inclusión de discursos representativos de diversos sectores de la sociedad y no sólo de aquellos que apelen a la ciudad letrada (Sánchez Prado, 2002: 265).
\end{abstract}

En tanto que son estos grupos no pertenecientes a la «ciudad letrada» los que se presentan como problema para el cuestionamiento del canon (no se dice qué grupos, cuántos, ni cuáles serían los criterios de selección), podemos decir que el concepto de canon de este autor no es sólo elitista, sino también clasista y racista, puesto que se acepta que el canon cambia de generación en generación, pero este cambio está marcado por las instituciones en las que se inscriben los intelectuales orgánicos (universidades, editoriales, instituciones políticas...) que son los que, en última instancia, tienen el poder y la palabra. Los grupos marginales, subalternos, con las teorías que pretenden dar cuenta de sus respectivas literaturas y culturas, son descartados en

${ }^{8}$ La idea de la «transculturación» procede del antropólogo cubano Fernando Ortiz, quien la formuló en 1940 en su libro Contrapunteo cubano del tabaco y del azúcar. Ortiz distinguía entre la «aculturación» y la «transculturación». La primera es el proceso por el cual una cultura dominada recibe pasivamente ciertos elementos de otra, por lo que en ella misma se presenta una cierta «deculturación». La «transculturación» es el proceso por el cual una cultura adquiere en forma creativa ciertos elementos de otra, es decir, a través de ciertos fenómenos de «deculturación» y otros de «neoculturación». La teoría fue desarrollada por Ángel Rama quien recoge sus ideas sobre el tema en La transculturación narrativa en América Latina (1982). Para Rama, en la teoría de Ortiz no se atendía suficientemente a los criterios literarios de selectividad y de invención que son propios de la «plasticidad cultural». En cuanto a la selectividad, no sólo se aplica según este crítico a la cultura extranjera, sino sobre todo a la propia. A su juicio, las principales operaciones que se efectúan en la transculturación son cuatro: pérdidas, selecciones, redescubrimientos e incorporaciones.

${ }^{9}$ El concepto de heterogeneidad se lo debemos a Antonio Cornejo Polar que lo expone ya en el texto «El indigenismo y las literaturas heterogéneas. Su doble estatuto sociocultural» (1977). Cornejo llama aquí literatura homogénea a la que es producida y leída, respectivamente, por escritores y un público del mismo estrato social: en este caso la producción literaria circula dentro de un solo espacio social y tiene un alto grado de homogeneidad, se trataría de una sociedad que se habla a sí misma; es el caso de la narrativa de Salazar Bondy, Ribeyro, Zavaleta en el Perú, y de Donoso y Edwards en Chile. «Caracteriza a las literaturas heterogéneas, en cambio, la duplicidad de los signos socioculturales de su proceso productivo: se trata, en síntesis, de un proceso que tiene, por lo menos, un elemento que no coincide con la filiación de los otros y crea, necesariamente, una zona de ambigüedad y de conflicto» (Cornejo Polar, 1977: 77). Las crónicas de la Conquista, la poesía melgariana, la literatura gauchesca y la negroide y la narrativa de lo real-maravilloso, serían diferentes ejemplos de literaturas heterogéneas. 
este juego en el que el investigador interviene gustosamente porque sólo puede participar un grupo de elegidos. La cuestión del canon queda de esta manera desvalorizada y desarmada frente a cualquier estrategia realmente innovadora ${ }^{10}$.

\section{REFERENCIAS BIBLIOGRÁFICAS}

BLOOM, H. (1994). El canon occidental. Trad. de Damián Alou. Barcelona: Anagrama, 1995.

CELLA, S. (1998). «Canon y otras cuestiones». En Dominios de la literatura: acerca del canon, S. Cella (comp.), 7-16. Buenos Aires: Losada.

- (comp.) (1998). Dominios de la literatura: acerca del canon. Buenos Aires: Losada.

CORNEJO POLAR, A. (1977). «El indigenismo y las literaturas heterogéneas: su doble estatuto sociocultural». Revista de Crítica Literaria Latinoamericana 7-8, 7-21.

DÍAZ MARTÍNEZ. M. (s.f.). «Intrahistoria abreviada del caso Padilla». En $<h t t p: / / w w w . l i t e r a t u r a . u s /$ padilla/diaz.html $>$ (consultado en agosto de 2006).

DOMÍNGUEZ MICHAEL, C. (1997). «Observaciones sobre el canon de Bloom». Vuelta (México) 247, 57-59.

FRANCO, J. (1981). «Tendencias y prioridades de los estudios literarios latinoamericanos». Escritura (Caracas) 11, enero-junio, 7-19.

- (1989). Plotting Women. Gender and Representation in México. New York: Columbia.

LIENHARD, M. (1991). La voz y su huella. Escritura y conflicto étnico-social en América Latina 1492-1988. Hannover: Eds. del Norte.

${ }^{10}$ Entre los estudios sobre el canon aparecidos en América Latina y el Caribe, no de carácter global o general sino dedicados a países o regiones concretas, destaca Un banquete canónico de Rafael Rojas (2000), que se centra en Cuba, en concreto en los autores cubanos que recoge Bloom como representativos del canon latinoamericano, seis de dieciocho en total: Nicolás Guillén, Alejo Carpentier, José Lezama Lima, Guillermo Cabrera Infante, Severo Sarduy y Reinaldo Arenas. La lucidez y capacidad crítica de Rojas destacan claramente frente a la adhesión de Sánchez-Prado a los presupuestos bloomianos. 
LÓPEZ DE ABIADA, J. M. y PÉREZ CINO, W. (2005). «El boom y el canon: ajustes para un arreglo». En Boom y postboom desde el nuevo milenio: impacto y recepción, J. M. López de Abiada y M. Morales Saravia (eds.). Madrid: Verbum.

MIGNOLO, W. (1991). «Los cánones y (más allá de) las fronteras culturales (o ¿de quién es el canon del que hablamos?)». En El canon literario, E. Sullá (ed.), 237-270. Madrid: Arco-Libros, 1998.

- (1994-1995). «Entre el canon y el corpus. Alternativas para los estudios literarios y culturales en y sobre América Latina». En Crítica literaria hoy. Entre las crisis y los cambios: un nuevo escenario, C. Rincón y P. Schumm (eds.), 23-36. Nuevo Texto Crítico 14/15 [monográfico].

ORTIZ, F. (1940). Contrapunteo cubano del tabaco y el azúcar (Advertencia de sus contrastes agrarios, económicos, históricos y sociales, su etnografía y su transculturación). La Habana: Universidad Central de las Villas. Introducción de Bronislaw Malinowski. Prólogo y Cronología de Julio Le Riverend en la edición de Caracas: Ayacucho, 1987. Edición española con Prólogo y Edición al cuidado de María Fernando Ortiz Herrera en Madrid: Cuba / España, 1999. Edición de Enrico Mario Santi en Madrid: Cátedra, 2002.

POBLETE, J. (2003). Critical Latin American and Latino Studies. Minneapolis: University of Minnesota Press.

- (2006). «Literatura, heterogeneidad y migrancia transnacional». Nueva Sociedad 201, 90-105 [número monográfico dedicado a «Cultura latina en Estados Unidos»]. En <http://www.nuso.org/upload/articulos/3313_1.pdf> (consultado en agosto de 2006).

- (coord.) (2005). «Dossier: Los Latino Americanos en una perspectiva global-hemisférica». Iberoamericana. América Latina-España-Portugal 17.

PULIDO, G. (1999). «Harold Bloom, el canon occidental y su repercusión en España». The Grove. Working on English Studies 6, 193-204.

RAMA, Á. (1982). Transculturación narrativa en América Latina. México: Siglo XXI.

- (1984). La ciudad letrada. Hannover: Eds. del Norte.

RINCÓN, C. (1978). El cambio en la noción de literatura y otros estudios de teoría y crítica latino-americana. Lima: Instituto Colombiano de Cultura. 
ROJAS, Rafael (2000). Un banquete canónico. México: FCE.

SÁNCHEZ-PRADO, I. M. (2002). El canon y sus formas: la reivindicación de Harold Bloom y sus lecturas hispanoamericanas. México: Secretaría de Cultura / Gobierno del Estado de Puebla.

SANDOVAL, A. y APARICIO, F. (eds.) (2005). Hibridismos culturales: la literatura y la cultura de los latinos en los Estados Unidos. Revista Iberoamericana LXXI, 212, julio-septiembre [monográfico].

SILVA, J. M. (2002). «El crepúsculo del canon: la (de)formación». Cyber Humanitatis (Universidad de Chile), 32 En <http://cyberhumanitatis.uchile.cl/> (consultado en agosto de 2006).

SOSNOWSKI, S. (1987). «Sobre la crítica de la literatura hispanoamericana: balance y perspectivas». Cuadernos Hispanoamericanos 447, 143-159.

ZANETTI, S. (1998). «Apuntes acerca del canon latinoamericano». En Dominios de la literatura: acerca del canon, S. Cella (comp.), 87-105. Buenos Aires: Losada. 